\title{
EGFR signalling as a negative regulator of Notch1 gene transcription and function in proliferating keratinocytes and cancer
}

\author{
Vihren Kolev, Anna Mandinova, Juan Guinea-Viniegra, Bing Hu, Karine Lefort, Chiara Lambertini, Victor Neel, \\ Reinhard Dummer, Erwin F. Wagner \& G. Paolo Dotto
}

Nat. Cell Biol. 10, 902-911 (2008); published online 6th July 2008

The version of this Article initially published lacked sufficient detail on how samples for western blotting were processed. Many experiments involved analysis of multiple proteins of similar molecular weight, making stripping and re-probing of blots often problematic. For this reason, our preferred approach was to analyse the various proteins by parallel gels/blots, with immunoblotting of $\gamma$-tubulin or actin being performed to ensure good quality of samples and absence of protein degradation before or after cell extract preparations. For all immunoblot analysis performed in this paper, equal loading and transfer of proteins were verified by poinceau $S$ staining of membranes. Specifically, the $\gamma$-tubulin blots shown in Figs 1c,d, 2a,e and 3b,c were from parallel gels, with comparable results being obtained by re-probing the blots. In Figs 1g, 2d and $6 \mathrm{~d}$, tubulin expression was assessed by stripping and re-probing blots. In each of these cases, results were confirmed by an independent experiment. In Fig. 5b, EGFR, c-Jun and actin blots were performed by sequential blotting of the same membrane, whereas p53 expression was assessed by a parallel gel/blot.

\section{ERK1/2-dependent phosphorylation and nuclear translocation of PKM2 promotes the Warburg effect}

\author{
Weiwei Yang, Yanhua Zheng, Yan Xia, Haitao Ji, Xiaomin Chen, Fang Guo, Costas A. Lyssiotis, Kenneth Aldape, \\ Lewis C. Cantley and Zhimin Lu
}

Nat. Cell Biol. 14, 1295-1304 (2012); published online 25th November 2012

In the print version of this Article, the phosphoinositide 3-kinase inhibitor is incorrectly written as "LY290042". It should read "LY294002". This is correct in the PDF and HTML versions of the Article. 